\title{
Review
}

\section{The Use of Lead Protection for Staff in Mobile Radiographic Settings: Can Staff Abandon Its Use?}

\author{
Carla Munoz, BRadMedImag (Hons) [Student]*; Euclid Seeram, PhD, MSc, BSc, FCAMRT; \\ John Mc Inerney, BSc (RAD) Hons. GDipHPE, GDipCTImaging \\ Department of Medical Imaging and Radiation Sciences, Monash University, Melbourne, Australia \\ *Corresponding author \\ Carla Munoz, BRadMedlmag (Hons) [Student] \\ Department of Medical Imaging and Radiation Sciences, Monash University, Melbourne,Australia; E-mail: carla.munoz@outlook.com.au
}

\section{Article information}

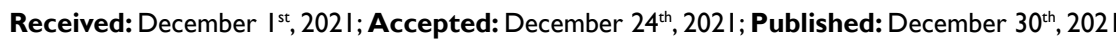

\section{Cite this article}

Munoz C, Seeram E, Mc Inerney J.The use of lead protection for staff in mobile radiographic settings: Can staff abandon its use? Radiol Open J. 202I; 5(I): 4-II. doi: I0. I7|40/ROJ-5-I34

\section{| ABSTRACT |}

The use of lead protection in patient contexts has recently come under scrutiny, with organisations moving towards ceasing its use in plain film radiography. The use of lead shielding in protecting staff in low-dose settings, such as plain film and mobile radiography, is therefore an important aspect of practice to be reviewed. The objective of this review is to evaluate the use of lead shielding in protecting staff in plain film and mobile radiography, while also exploring the evolution of occupational doses and perceptions of lead protection. While literature is limited on the use of lead protection for staff in mobile settings, lead protection has been shown to reduce staff and adjacent patient dose from scatter. Furthermore, despite the increased frequency of medical imaging procedures in contemporary radiography, the occupational doses of medical radiation workers have reduced over time. With literature demonstrating gaps in current understandings of radiobiological mechanisms at low doses, the linear no-threshold model utilised to estimate radiation risk and develop protection standards cannot be rejected. Thus, this review finds the need for further research to be undertaken to improve risk estimates at low doses in larger cohorts of medical radiation workers, for the demonstration of long-term effects from occupational exposure, prior to ceasing staff lead protection.

Keywords

Radiation protection; Occupational dose; Lead protection; Mobile radiography; Radiography; COVID-19 pandemic.

\section{INTRODUCTION}

S oon after the initial discovery and use of X-rays as a therapeutic and diagnostic tool, the emergence of acute adverse health effects prompted the need for radiation protection measures. ${ }^{1}$ The innovation of lead protection arose in the late 1800 s to early $1900 \mathrm{~s}$, majorly from contributions by William Rollins, an early pioneer of radiation protection. ${ }^{1}$ Furthermore, with the initial establishment of the International Commission on Radiological Protection (ICRP), previously known as the International X-ray and Radium Protection Committee in 1928, formal radiation protection standards began to be devised. ${ }^{2}$ Nuclear warfare during World War II fast-tracked the establishment of radiation protection standards, prompting innovations in radiation detection instrumentation and radiobiological research. ${ }^{1}$ Both public and occupational radiation limits were introduced as protective measures against emerging somatic effects, such as primary solid tumours and leukaemia. ${ }^{2}$ Extended epidemiological follow-up of atomic bomb survivors demonstrated increased radiation-induced risks for leukaemia, with subtype effect differences influenced by age at exposure, sex, and environmental risk. ${ }^{3}$ Hence, the need to limit potential radiation-induced effects has been a central pillar in the protection of patients, staff and the public in the development of modern radiology. Justification of radiation usage, optimisation of protection and the application of dose limits have remained the fundamental principles of protection to prevent the induction of tissue reactions and reduce the risk of stochastic effects. ${ }^{4}$

Despite advances in medical imaging procedures and techniques increasing the frequency of procedures over the past few decades, trends in the occupational exposure of radiologists and radiographers have shown decline. ${ }^{5}$ Although this could possibly be attributed to the use of shielding, lead protection for patients has recently come under scrutiny within the realm of plain film imaging. Specifically, the use of lead shielding has come under scrutiny in protecting patients in pelvic examinations, with discus- 
sions suggesting shielding introduces more risks than benefits to patients. ${ }^{6-8}$ In the backdrop of a reduction in reproductive organ tissue weighting factors from 0.20 in $1990^{\circ}$ to 0.08 in $2007,{ }^{4}$ contemporary perceptions advocating for the abandonment of gonadal shielding may have been fostered. Nevertheless, with current trends advocating for the ceased use of gonadal and foetal lead protection for patients in plain film imaging, ${ }^{10-13}$ equal consideration should also be given to whether staff might cease the use of lead protection if its use is deemed inconsequential for patients.

At present, published literature focuses on shielding in high-dose imaging environments, such as fluoroscopy or the operating theatre. ${ }^{14-17}$ However, with support increasing for the discontinued use of patient lead shielding, it is imperative that all aspects of this discussion are considered to ensure continued safety, prior to the formulation of a consensus. This is of particular importance if current perceptions or beliefs regarding shielding negatively influence the compliance of staff in using self-protection or patient protection in different radiographic settings without sufficient scientific backing. While variability may naturally exist in individuals' practice, it is imperative that medical radiation practitioners wholly comprehend the risks and benefits of abandoning lead shielding prior to enacting these behaviours in practice.

With the development of new techniques in the face of exceptional circumstances presented by the coronavirus disease-2019 (COVID-19) pandemic, the demand for mobile imaging has increased. Staff have the potential to be left without shielding and may be limited by achievable distance from radiation sources or patients being imaged. Thus, a current review of the use of lead shielding in staff safety, particularly in mobile imaging contexts, is timely.

This literature review will examine the use of lead protection in plain film radiography with a focus on staff safety in mobile radiographic settings. It will also explore the history of lead protection and occupational doses, as well as evolving perceptions surrounding its use.

\section{METHOD}

A review was undertaken to examine the topic through existing literature, with an exploratory search of Monash University Library Search, PubMed, Ovid MEDLINE and Google Scholar conducted between August and October 2021. Various key words, including "historical development", "history", "evolution", "lead", "protection", "shielding", "personal", "garment", "apron", "mobile", "portable", "bedside", "radiography", "ICU”, "medical radiation worker", "radiographer", "radiologic technologist", "staff”, "radiation" and "occupational exposure" were utilised to identify relevant articles for review. Where suitable, keywords were combined with Boolean operators (and/or) for search. Alongside this, an inspection of the reference sections of the most significant and relevant articles was performed to expand the breadth of investigation, particularly for analysis of frequently-appearing citations. Articles were filtered by year depending on topic for relevance, with articles relevant for historical data having a wider scope of search (1980 onwards) than lead shielding studies (which were limited to
2014 for relevance). Further inclusion criteria included Englishlanguage publishing and full-text peer reviewed articles. Searches of various relevant organisational body's documents was also undertaken. Abstracts of articles were read to analyse and ensure relevance to the topic, followed by in-depth analysis of those deemed pertinent to the topic.

\section{DISCUSSION}

\section{A Brief History of Radiation Protection and the Evolution of its Perceptions}

With the emergence of acute radiation injuries after the discovery of X-rays, the need to protect against overexposure was recognised and early developments were made into what became known as radiation protection measures. ${ }^{1,2}$ William Rollins, an American dentist by trade, contributed greatly to the early development of lead protection, suggesting the use of lead walls and goggles, as well as protective tube housing and collimators in the late $1800 \mathrm{~s}$ and 1900s. ${ }^{1,18}$ Literature describes lead shielding being applied in routine practice as early as 1905 for patients in gonadal form, to prevent male sterility, as had been seen in animal models and male medical radiation workers. ${ }^{7}$ However, limited literature exists on the precise implementation of shielding for medical radiation workers. Early models demonstrate protection from radiation emitted from hand-held fluoroscopes, intended to reduce radiation-induced dermatitis. ${ }^{1}$ However, with limited knowledge of scatter radiation, initial methods of lead shielding such as these did not provide adequate protection.

Public perceptions of radiation also influenced the early development of protection efforts. These perceptions were shaped by knowledge of legal action taken by those adversely affected by the inappropriate use of diagnostic X-rays, alongside the radiationinduced deaths of medical radiation workers in Europe. ${ }^{1,18}$ The establishment of early radiation protection bodies in Britain (the International X-ray and Radium Protection Committee) and America (the US Advisory Committee on X-ray and Radium Protection) were driven by these events, with early recommendations published by both committees in 1921 and 1922, respectively. ${ }^{18}$ Early British recommendations advocated for limited work times and annual holidays away from radiation sources for medical radiation workers. ${ }^{19}$ Over time, these limitations became adapted to effective doses, standardising the exposure limits proposed for radiation staff and the public. $^{20}$

Years later, the advent of nuclear warfare demonstrated detrimental health effects associated with high radiation doses and sparked significant concerns for the possible genetic effects of radiation, leading protection bodies to implement occupational whole-body and public dose limitations. ${ }^{2}$ The radiation principle of maintaining exposures "as low as reasonably achievable" arose from this cautionary mindset post-World War II, as it was unknown whether a threshold existed for health effects to occur. ${ }^{2}$ With the limited epidemiological follow-up of atomic bomb survivors providing much of the radiobiological information forming the basis of early genetic and hereditary risk concepts, gonadal shielding was initially supported. ${ }^{7}$ However, with advances in the understanding 
of genetic risk and reproductive organ susceptibility, the tissue weighting factor used to calculate effective dose for the gonads was reduced from 0.20 in $1990^{\circ}$ to 0.08 in $2007 .^{4}$ This reduction in tissue weighting factor has impacted practice towards the abandonment of gonadal shielding for patients. ${ }^{12,13}$ However, simultaneously within this period, the tissue weighting factor for the breast observed an increase from 0.05 in $1990^{\circ}$ to 0.12 in $2007 .{ }^{4}$ As such, due consideration should also be given to the observed increase in breast tissue weighting. If the use of lead protection is ultimately deemed inconsequential for patients in a plain film context, an equal consideration for staff should be undertaken. Thus, it is important to fully determine specific situations in which shielding may or may not be necessary, in gonadal or breast contexts, prior to establishing a firm stance on the abandonment of lead protection. Moreover, a recent study demonstrates the potential for increased risk of testicular germ cell cancer for patients exposed to diagnostic radiation from 0-10-years of age, compared to those exposed at or after 18-years of age. ${ }^{21}$ This further highlights the need for evidence to drive any abandonment practices, particularly when reported radiobiological tissue weightings have not been updated in over a decade and risk may differ between males and females.

\section{Occupational Radiation Exposures and Risk Modelling}

With increasing knowledge of radiation and advancing protection techniques, occupational dose limits for medical radiation workers fell from early-levels of $0.6 \mathrm{~Sv} /$ year in the $1900 \mathrm{~s}-1930 \mathrm{~s}$ to $50 \mathrm{mSv} /$ year in $1958 .^{2}$ At present, the Australian Radiation Protection and Nuclear Safety Agency (ARPANSA) limits workers to an occupational exposure of $20 \mathrm{mSv}$ per year, averaged over 5 consecutive years. ${ }^{22}$ These limitations aim to protect medical radiation workers from excessive exposure and acute radiation effects, as evident in early radiation practice.

Technological advances have allowed for the development of high-dose imaging procedures and greater patient throughput, increasing the contribution of medical imaging to the radiation dose of the population, estimated at approximately $1.7 \mathrm{mSv}$ in 2010 in Australia. ${ }^{23}$ Despite this, the annual average occupational effective dose of radiologists and radiographers has reduced over time. ${ }^{5}$ The annual effective radiation doses fell from approximately $5 \mathrm{mSv}$ in the 1960 s to less than $1 \mathrm{mSv}$ in the $1990 \mathrm{~s}$, with dose levels continuing to fall in the $2000 \mathrm{~s}^{5}$ Although this could infer protection measures can positively contribute to reduced doses over time, the use of lead aprons or other shielding apparatus has not been outwardly specified in reporting. ${ }^{5}$ This is problematic as the true nature of lead shielding in protecting medical radiation workers is not able to be properly quantified without adequate delineation of protective practices in relation to reported doses. However, it has been noted that the use of lead aprons in low energy X-ray fluoroscopy settings increases the complexity of determining the absorbed doses of shielded torso organs when dosimeters are used externally to shielding. ${ }^{24}$ Nonetheless, improved staff and patient protection was noted with the use of personal radioprotective equipment, with most torso organs associated with stochastic risk being shielded by lead aprons. ${ }^{20,24}$

The analysis and evaluation of occupational dose pat- terns and trends over time provides great insight into how wellprotection practices safeguard staff against radiation, particularly when changes are implemented. In order to determine accurate outcomes, occupational dose data should be of high quality and quantity. Although historical reviews of medical radiation workers' occupational doses demonstrate reduction over time, data is limited by insufficient follow-up, inconsistencies in dosimetry measurement and lack of information regarding worker lifestyle and environmental factors. ${ }^{25}$ These factors are crucial in bettering the understanding of adverse health effects from low-dose exposures, particularly in populations of radiation workers.

Alongside this, projection studies demonstrate the potential for female diagnostic radiation workers to exhibit higher projected lifetime attributable radiation risk than males, with an overall estimated increased risk of colon and thyroid cancer in all diagnostic radiation workers. ${ }^{26}$ Furthermore, significant dose-response relationships for breast cancer mortality have been observed in a cohort of US radiographers, particularly those employed prior to 1950. ${ }^{27}$ Hence, with the knowledge that medical radiation workers are at increased risk of colon, thyroid and breast cancer, it is imperative to determine how well current shielding protects these body areas for staff, as these areas would ideally be shielded by radiation with correct adherence to lead protection practices.

The linear no-threshold model: While many biological and epidemiological studies of dose provide the basis for radiation protection standards and occupational dose limits, it is important to acknowledge the strengths and shortcomings of the linear no-threshold (LNT) model used to develop risk projections and protection practices. The LNT model was adopted in low dose settings through the extrapolation of data from follow-up studies conducted on atomic bomb survivors and assumes radiation risk increases proportionally with dose, without a specific threshold for effect induction. ${ }^{28}$ Although this model provides useful risk estimates at high doses, estimates at low doses are challenging to accurately establish due to data limitations, difficulty in separating true risk from background radiation risk and the presence of various confounders at low doses. ${ }^{28,29}$ It is important to note that at low doses, individual radiosensitivity, genetic variations, variations in baseline disease incidence for different populations, socioeconomic, lifestyle or environmental factors - such as background radiation and pollutants - can all confound and distort results. ${ }^{28}$ Furthermore, the basis of low-dose modelling being adapted from high dose exposure cohorts may be inaccurate for low-dose risk assessment.

To improve risk estimate models and statistical significance of low dose epidemiological study results, large sample sizes are required with complete lifetime follow-up, making studies difficult to achieve. ${ }^{28}$ However, the current National Council on Radiation Protection (NCRP)'s Million Person Study (MPS) underway aims to address the shortcomings of older epidemiological studies and improve risk estimate modelling at low doses, assessing health effects in a large cohort of US radiation workers and veterans exposed to low doses. ${ }^{30}$ The results of the MPS and similar studies in the future would provide further insight into current knowledge gaps of health effects at low doses and support or refute the valid- 
settings in contemporary studies.

ity of the LNT model in developing radiation protection practice and risk modelling. Hence, updated knowledge on LNT modelling at low doses will truly solidify whether lead shielding can be abandoned by staff in mobile settings. However, given the current limitations on risk estimate modelling, the LNT model is regarded as the most appropriate model for determining risk at low doses, although it may not correspond to true risk. ${ }^{28}$ With the potential for adaptive radiobiological responses to affect the validity of the LNT model, ${ }^{28}$ further research is required to improve current risk models and radiobiological responses, with large cohort investigations accounting for responses and variations in individual factors.

\section{Lead Protection in Mobile Radiography}

Although there has been limited research conducted on staff doses and lead protection within mobile imaging settings, literature demonstrates the ability of lead shielding to reduce dose from scatter. Early dose quantification studies conducted in mobile settings allowed for improved understandings of mobile scatter distributions and the safety of adjacent patients. ${ }^{31} \mathrm{~A}$ dose quantification study demonstrated that staff doses from portable radiographs performed within a typical emergency department setting were minimal, supporting protection measures of shielding when distance is not achievable. ${ }^{32}$ Alongside this, radiographers were found to receive higher doses than emergency physicians. ${ }^{33}$

More recent investigations of scatter from mobile phantom studies have also observed a reduction in dose from scatter with the implementation of shielding, even when adequate distance is maintained from the primary beam. ${ }^{34}$ Additionally, a study conducted in a neonatal intensive care unit (NICU) has demonstrated a reduction in scattered dose to not only staff, but adjacent patients and caregivers when shielding is utilised in mobile radiography - with adjacent patients having dose reduced by an approximate factor of $100 .{ }^{35}$ Despite this, the authors do not support use of lead shielding and anti-X sheets in NICU mobile imaging due to potential interference with adjacent patient monitoring equipment if placed on incubators, alongside low maximal scatter doses for staff and caregivers. Hence, it may be prudent to acknowledge that although lead shielding does demonstrate dose reduction benefits for individuals near the patient imaged in mobile NICU settings, using shielding sheets to reduce doses may not be suitable if $\mathrm{pa}$ tient monitoring is hindered.

Investigations of scattered doses imparted on adult populations due to mobile imaging demonstrate consistent results with NICU, with intensive care unit (ICU) staff receiving lower exposures than stipulated reference levels, at $<0.6 \mathrm{mSv} /$ year. $^{36}$ Thus, with very low scatter radiation doses observed in mobile imaging contexts each year with and without shielding, it may be conceivable that lead shielding can be mitigated in a mobile context. However, with the potential for increased breast cancer risk in medical radiation workers, it is worthwhile for workers at a minimum, to continue utilising individual lead protection. As aforementioned, further advances in radiobiological models of cancer induction at low dose levels are required prior to reaching unanimity in the discontinuation of lead protection for staff. It is apparent that seldom focus is placed on lead protection reducing staff dose in mobile

\section{Lead Protection in Plain Film Radiography}

The benefits of lead protection have been established in plain film radiography, within primary X-ray beam contexts. An experimental study determined significant scatter dose reductions to patient's breasts and eyes when lead shielding was implemented for typical examination of the cervical spine, including anteroposterior (AP) and left lateral projections. ${ }^{37}$ Specifically, breast dose was reduced by $99.9 \%$ in the AP projection, with left and right breast dose reduced by 23 and $99 \%$ in the lateral projection. Consequently, left and right eye doses were reduced by 91 and $89 \%$ in the AP projection, and 23 and $99 \%$, respectively, for the lateral projection. Despite establishing the effectiveness of shielding in protecting radiosensitive organs exposed to scatter in a patient context, the study is limited by its use of an anthropomorphic phantom and cannot account for variations in patient size.

Currently, there is very limited literature available on doses imparted to helpers - which may consist of staff or carers - in plain film examinations. A study conducted in an Australian paediatric hospital concluded that helpers remaining in the room to assist with emotional comfort or positioning in plain film paediatric examinations do not require lead protection when scattered dose to the carer is $<2 \mu \mathrm{Sv}^{38}$ However, the study was limited by its use of a self-determined arbitrary dose threshold of $2 \mu \mathrm{Sv}$, its inability to account for examinations with multiple projections or repeat imaging and its baseline dose comparison to background radiation levels. As such, it is impractical to base decisions of lead protection discontinuation on arbitrary dose thresholds, despite being below stipulated standards of $1 \mathrm{mSv} /$ year for members of the general public. $^{22}$ Discontinuation of lead protection in this sense does not align with protective policies developed based on the LNT model of risk. Thus, it is imperative that future research focuses on accurate models of risk, accounting for adaptive processes, to ensure protective practices can be accurately updated against the current shortcomings of the LNT model in low-dose settings. At present, this data is not available and as such, abandonment practices are not appropriate for carers/helpers under current policies and understandings.

Additionally, observations that lead aprons may increase the absorbed dose to the thyroid if worn without a thyroid collar in patient settings ${ }^{39}$ may generate cause for concern for staff if they do not supply complete protective attire for patients.

Potential risks to staff in using lead protection: With the potential for lead to cause adverse health effects in and of itself, the finding that lead shielding can expose healthcare workers to lead dust may introduce risks associated with frequent use. Findings demonstrate higher lead concentration in radiographers' hair compared to administration staff due to the use of lead protection and detectable lead dust on shields that are improperly stored. ${ }^{40,41}$ However, an analysis of radiology staff's blood lead levels demonstrated staff were unlikely to suffer from lead poisoning with frequent use of personal shielding. ${ }^{42}$ Thus, with correct hanged storage and frequent quality control of lead garments, the potential risk of lead 
exposure to staff can be reduced in individual departments while maintaining radiation protection standards. Nonetheless, the possibility exists that this notion may drive the profession to utilise alternative lead-equivalent materials in shielding to prevent lead dust exposure in future if further investigation demonstrates significant health risks to staff.

\section{Staff Perceptions towards Lead Protection in Practice}

As medical radiation workers are at increased risk of colon, thyroid and breast cancers, it is imperative to determine how well-staff adhere to self-protection practices as coverage of these areas would be upheld with the appropriate use of radioprotective garments. Studies demonstrate mixed staff adherence to personal radiation protection practices. ${ }^{43,44}$ In a cohort of United Arab Emirates (UAE) radiographers, personal radiation protection adherence was found to be significantly higher in older, more experienced groups of radiographers. ${ }^{43}$ However, qualification level was not determined to be a factor affecting self-protection. Moreover, studies into the adherence of lead apron usage demonstrate radiographers in South Korea utilising radioprotective garments $50.3 \%$ of the time when exposed to radiation, with higher adherence in general hospital settings. ${ }^{45}$ Contrastingly, this study demonstrated medical radiation workers employed for less than 10-years as exhibiting higher radioprotective garment adherence practices than more experienced groups. However, this may be affected by a disparity between less experienced participants surveyed (62.4\%), compared to more experienced participants $(37.6 \%)$. It is also important to acknowledge that these studies focus on varying levels of exposure in different radiology environments - not only plain film or mobile imaging. As there is limited literature available on mobile shielding in practice, an evaluation of staff perceptions towards lead shielding in a general sense enables a broader picture to be detailed.

Furthermore, issues with hygiene and equipment availability have also been identified in literature as factors negatively influencing self-protection practices, with staff less likely to use thyroid shields due to availability and garment cleanliness. ${ }^{44} \mathrm{Di}$ rect addressing of these factors in individual departments with increased sanitisation processes for radioprotective garments would therefore positively influence usage.

It is also important to consider how radiographer's attitudes and perceptions have changed towards lead protection in the sense of general practice, alongside self-adherence. An ethnographic study of radiographers' attitudes and perceptions towards lead protection in the United Kingdom suggests personal beliefs and values are beginning to influence disparities in the clinical application of lead protection for patients. ${ }^{46}$ The ceased use of lead protection in practice by some radiographers was identified to stem from word of mouth, rather than evidence-based literature, presenting concern for arising disparities in clinical decision-making. Although variations may naturally exist in individual medical radiation workers' practice, it is imperative for practitioners to fully understand the risks and benefits of abandoning lead protection in their individual clinical and self-protection practices and make these decisions from evidence - the ultimate basis for clinical decision-making.

\section{Exceptional Circumstances in Mobile Settings Coronavirus Disease-2019}

With the advent of the COVID-19 pandemic presenting new challenges in healthcare, exceptional circumstances have prompted the development of new techniques in mobile imaging. The 'throughglass' technique has been independently implemented in various radiology departments globally to combat the spread of COVID-19, reduce waste from personal protective equipment (PPE) usage and supplement the increased demand of mobile imaging. ${ }^{47-49}$ However, with the implementation of this technique, healthcare staff have the potential to be left in the room without shielding and with limited distance achievable from the radiation source or the patient being imaged. Thus, ensuring the safety of staff in exceptional mobile circumstances is imperative, particularly when the use of lead shielding has come under scrutiny.

Through viability testing of the through-glass technique, literature indicates that with a variety of technical factors used, 'through-glass' mobile imaging conditions are safe for staff to operate without lead shielding while remaining at an appropriate distance. ${ }^{49}$ Despite low dose scatter measurements reported at increased distances $(3 \mathrm{~m})$ compared to conventional mobile settings, authors suggest the continued use of lead protection for practitioners remaining within the room and for an increased safety distance of 3-4 $\mathrm{m}$ for surrounding staff and patients. ${ }^{48}$ Furthermore, the use of easily cleanable and portable shielding has been proposed for staff remaining in the patient's room to reduce additional exposure for staff who are already 'dirty' within the room and unable to don personal lead shielding. ${ }^{47}$

In accordance with the LNT model for radiation risk, as many protective measures as possible - including time, distance and shielding - should be undertaken by staff even in low-dose radiation environments, as data at present does not suggest alternate risk modelling to estimate adverse health effects. ${ }^{45}$ Hence, even in exceptional circumstances in mobile settings, lead protection can provide practical radiation safety for staff unable to exit the patient room during imaging and contribute to a reduced occupational dose.

\section{What does this Mean for Our Professional Responsibilities?}

As the COVID-19 pandemic has demonstrated how quickly healthcare practice and societal norms can be adapted to overcome universal challenges, it is possible that professional responsibilities may also be modified in the backdrop of the pandemic. For instance, current Australian Professional Capabilities for Practice describe qualified radiographers necessitating the ability to undertake interprofessional collaboration to be fit to practice. ${ }^{50}$ However, in the exceptional circumstances stimulated by COVID-19, radiographers have been required to collaborate closely with nurses, coaching them in some instances to achieve optimal imaging receptor positioning for 'through-glass' techniques. Hence, there is potential for the expansion of healthcare workers' capabilities into radiation safety practices as increased collaboration is apparent with the introduction of new, highly infectious viruses. Thus, a set of alternate capabilities may be required of healthcare workers, which may 
apply to future pandemics, for enhanced collaboration and breadth into radiation protection practices.

\section{LIMITATIONS}

As alluded to, epidemiological studies of occupational dose and risk estimate are limited by short follow-up periods that do not allow for visualisation of cancer development in older-aged workers. ${ }^{5,25-27}$ Alongside this, epidemiological studies conducted for low dose exposures are subject to many confounding variables and lack sufficient consideration for lifestyle and environmental factors that may distort risk estimates. Furthermore, low dose risk estimates based on the LNT model are limited by shortcomings in extrapolating high dose settings to low doses. ${ }^{28-30}$ Further epidemiological research studies conducted with larger cohorts and entire lifetime follow-up would enable better understanding of current knowledge gaps concerning the validity of the LNT model at low doses. Consequently, a focus on advancing knowledge gaps in adaptive radiobiological responses may provide further insight into the true validity of current risk estimates.

Moreover, is apparent that literature regarding the use of lead shielding in protecting staff in mobile settings is severely limited. Of published literature, studies investigating the use of lead shielding are limited by anthropomorphic phantom usage, which do not account for variations in patient size and/or composition and may result in altered scatter distributions in mobile settings. ${ }^{34,48,49}$ However, this is an unavoidable limitation, as it is unethical to irradiate patients solely for dose quantification to staff, unless patients already undergoing medical imaging procedures are consented and recruited. Furthermore, studies conducted in neonatal populations are limited in generalisability, due to larger doses being used for adult portable imaging, which may alter scatter distributions. ${ }^{35}$ Moreover, a study conducted in an adult ICU was majorly limited by its minimal description of resultant staff dose measurements, with no data analysis or tabulations performed. ${ }^{36}$

Furthermore, studies focussing on the perceptions and attitudes of staff towards personal adherence to lead protection are restricted by their small sample sizes and collection of responses through self-administered surveys, with potential uncertainty arising from individual's responses. ${ }^{43,46}$ Alongside this, one study was potentially influenced by selection bias with departmental nomination of particular participants for inclusion in the study, who may have exhibited higher adherence to lead protection. ${ }^{44}$

Considering hereditary risk concepts have not been formally updated since a reported reduced gonadal sensitivity in 2007, proposal of lead abandonment due to this metric is not optimal. In order to fully negate or support the use of lead protection, an update of tissue weighting metrics should be undertaken with the most recent data available for low-dose cohorts, even if this update solidifies no changes to current tissue weighting values. However, this limitation may be resolved within the coming years, as the ICRP are in the process of revising recommendations for modern practice. ${ }^{51}$

\section{CONCLUSION}

Presently, it cannot be concluded that staff should abandon lead shielding in the low-dose settings of mobile and plain film radiography, although there is movement towards abandonment in patient contexts. Further research is required to achieve true unanimity in the cessation of lead protection for staff in mobile radiography settings. Specifically, further research into current gaps in radiobiological understandings will facilitate better comprehension of adaptive mechanisms present at low doses. Alongside this, updating risk estimation models with large cohort studies will enable confidence in or refute the relevance of the LNT model in current cancer and solid tumour risk estimates for contemporary low-dose exposures experienced by medical radiation workers. With this knowledge, it would be possible to accurately examine the necessity of lead protection in protecting and reducing staff dose in low-dose radiography. At present, the LNT model is considered the most appropriate for low-dose risk estimates and for the development of radiation protection practices. Hence, it is impractical to base staff abandonment of lead shielding upon reduced radiosensitivity tissue weightings as current modelling assumes there no threshold for inducing health effects. Thus, even in low-dose settings of plain film and mobile radiography, lead abandonment cannot be purported, particularly when studies demonstrate reduction in scatter from staff shielding. Differences in staff perceptions and attitudes towards the personal use of lead protection must also be adequately addressed in the profession, as studies indicate decisions are being made on information received as word of mouth, rather than evidence. Furthermore, potential health implications of using lead shielding must be further investigated for the future of the profession if lead shielding is to be maintained for staff in low-dose settings. However, the potential implementation of lead-equivalent materials may be useful in protecting staff if lead exposure is determined to be a significant health risk. Thus, as lead protection provides notable dose reductions for staff in low-dose settings and current modelling does not suggest otherwise, staff shielding should not be abandoned in contemporary practice until low-dose exposure understandings are improved.

\section{CONFLICTS OF INTEREST}

The authors declare that they have no conflicts of interest.

\section{REFERENCES}

1. Brodsky A, Kathren RL. Historical development of radiation safety practices in radiology. Radiographics. 1989; 9: 1267-1275. doi: 10.1148/radiographics.9.6.2685944

2. Edwards M. Development of radiation protection standards. Radiographics. 1991; 11: 699-712. doi: 10.1148/radiographics.11.4.1887122

3. Preston DL, Kusumi S, Tomonaga M, et al. Cancer incidence in atomic bomb survivors. Part III: Leukemia, Lymphoma and multiple myeloma, 1950-1987. Radiat Res. 1994; 137: S68-S97. doi: $10.2307 / 3578893$ 
4. No authors listed. The 2007 recommendations of the international commission on radiological protection. Ann ICRP. 2007; 37(2-4): 1-332. doi: 10.1016/j.icrp.2007.10.003

5. Linet MS, Kim KP, Miller DL, Kleinerman RA, Simon SL, Berrington de Gonzalez A. Historical Rreview of occupational exposures and cancer risks in medical radiation workers. Radiat Res. 2010; 174: 793-808. doi: 10.1667/RR2014.1

6. Warlow T, Walker-Birch P, Cosson P. Gonad shielding in paediatric pelvic radiography: Effectiveness and practice. Radiography. 2014; 20: 178-182. doi: 10.1016/j.radi.2014.01.002

7. Jeukens CRLPN, Kutterer G, Kicken PJ, et al. Gonad shielding in pelvic radiography: Modern optimised X-ray systems might allow its discontinuation. Insights Imaging. 2020; 11: 15. doi: 10.1186/ s13244-019-0828-1

8. Winfeld M, Strubel N, Pinkney L, et al. Relative distribution of pertinent findings on portable neonatal abdominal radiographs: Can we shield the gonads? Pediatr Radiol. 2013; 43: 1295-1302. doi: $10.1007 / \mathrm{s} 00247-013-2700-7$

9. No authors listed. 1990 recommendations of the international commission on radiological protection. Ann ICRP. 1991; 21(1-3): $1-201$.

10. Australian Society of Medical Imaging and Radiation Therapy (ASMIRT). ASMIRT Position Statement: Gonadal Shielding. Victoria, Australia 2020: 3.

11. The American Association of Physicists in Medicine (AAPM). AAPM Position Statement on the Use of Patient Gonadal and Fetal Shielding. Alexandria, VA, USA: AAPM; 2019.

12. The British Insititute of Radiology (BIR). Guidance on Using Shielding on Patients for Diagnostic Radiology Application. London, UK: The British Institute of Radiology (BIR); 2020: 1-87.

13. Marsh RM, Silosky M. Patient Shielding in diagnostic imaging: Discontinuing a legacy practice. AJR Am J Roentgenol. 2019; 212: 755-757. doi: 10.2214/AJR.18.20508

14. Cheon BK, Kim CL, Kim KR, et al. Radiation safety: A focus on lead aprons and thyroid shields in interventional pain management. Korean J Pain. 2018; 31: 244-252. doi: 10.3344/kjp.2018.31.4.244

15. Tonkopi E, Lightfoot C, LeBlanc E. SU-F-I-72: Evaluation of the ancillary lead shielding for optimizing radiation protection in the interventional radiology department. Medical Physics (Lancaster). 2016; 43: 3403-3403. doi: 10.1118/1.4955900

16. Hu P, Kong Y, Chen B, Liu Q, Zhuo W, Liu H. Shielding effect of lead glasses on radiologists' eye lens exposure in interventional procedures. Radiat Prot Dosimetry. 2017; 174: 136-140. doi: $10.1093 / \mathrm{rpd} / \mathrm{ncw} 098$

17. Shortt CP, Al-Hashimi H, Malone L, Lee MJ. Staff radiation doses to the lower extremities in interventional radiology. Cardiovasc Intervent Radiol. 2007; 30: 1206-1209. doi: 10.1007/s00270-0079071-0

18. McGinley P, Miner M. A history of radiation shielding of X-ray therapy rooms. Health Phys. 1995; 69: 759-765. doi: 10.1097/00004032-199511000-00010

19. Rolleston H. X-ray and radium protection Committee. Radiology. 1924; 3: 171-176. doi: 10.1148/3.2.171b

20. Boice J, Dauer LT, Kase KR, Mettler FA, Vetter RJ. Evolution of radiation protection for medical workers. Br J Radiol. 2020; 93 : 20200282. doi: 10.1259/bjr.20200282

21. Nead KT, Mitra N, Weathers B, et al. Lower abdominal and pelvic radiation and testicular germ cell tumor risk. PLoS One. 2020; 15: e0239321. doi: 10.1371/journal.pone.0239321

22. Australian Radiation Protection And Nuclear Safety Agency (ARPANSA). Code for Radiation Protection in Planned Exposure Situations. 2020. Web site. https://www.arpansa.gov.au/sites/default/files/rps_c-1_rev_1.pdf. Accessed November 29, 2021.

23. Hayton A, Wallace A, Marks P, Edmonds K, Tingey D, Johnston P. Australian per caput dose from diagnostic imaging and nuclear medicine. Radiat Prot Dosimetry. 2013; 156: 445-450. doi: $10.1093 / \mathrm{rpd} / \mathrm{nct} 101$

24. Yoder CR, Dauer L, Balter S, et al. Dosimetry for the study of medical radiation workers with a focus on the mean absorbed dose to the lung, brain and other organs. Int J Radiat Biol. 2018; 1-36. doi: 10.1080/09553002.2018.1549756

25. Chartier H, Fassier P, Leuraud K, et al. Occupational low-dose irradiation and cancer risk among medical radiation workers. Occup Med (Oxford). 2020; 70: 476-484. doi: 10.1093/occmed/kqaa130

26. Lee WJ, Choi Y, Ko S, et al. Projected lifetime cancer risks from occupational radiation exposure among diagnostic medical radiation workers in South Korea. BMC Cancer. 2018; 18: 1206. doi: 10.1186/s12885-018-5107-x

27. Preston DL, Kitahara CM, Freedman DM, et al. Breast cancer risk and protracted low-to-moderate dose occupational radiation exposure in the US Radiologic Technologists Cohort, 1983-2008. BrJ Cancer. 2016; 115: 1105-1112. doi: 10.1038/bjc.2016.292

28. Seong KM, Seo S, Dalnim L, et al. Is the linear no-threshold dose-response paradigm still necessary for the assessment of health effects of low dose radiation? J Korean Med Sci. 2016; 31: S10-S23. doi: 10.3346/jkms.2016.31.S1.S10

29. Shore RE, Beck HL, Boice JD, et al. Implications of recent epidemiologic studies for the linear nonthreshold model and radiation protection. J Radiol Prot. 2018; 38: 1217-1233. doi: 10.1088/13616498/aad348 
30. Boice JJD, Cohen SS, Mumma MT, Ellis ED. The million person study, whence it came and why. Int J Radiat Biol. 2019; 1-14. doi: 10.1080/09553002.2019.1589015

31. Trout E, Kelley J, Kellner J. Scattered radiation from bedside Roentgen examinations. Am J Roentgenol Radium Ther Nucl Med. 1972; 114: 832-836. doi: 10.2214/ajr.114.4.832

32. Grazer RE, Meislin HW, Westerman BR, Criss EA. A nine-year evaluation of emergency department personnel exposure to ionizing radiation. Ann Emerg Med. 1987; 16: 340-342. doi: 10.1016/ s0196-0644(87)80183-x

33. Braun BJ, Skiendzielewski JJ. Radiation exposure of emergency physicians. Ann Emerg Med. 1982; 11: 535-540. doi: 10.1016/s01960644(82)80426-5

34. Chiang H-W, Liu Y-L, Chen T-R, Chen C-L, Chiang H-J, Chao $\mathrm{S}-\mathrm{Y}$. Scattered radiation doses absorbed by technicians at different distances from X-ray exposure: Experiments on prosthesis. Biomed Mater Eng. 2015; 26: S1641-S1650. doi: 10.3233/BME-151463

35. Longo M, Genovese E, Donatiello S, et al. Quantification of scatter radiation from radiographic procedures in a neonatal intensive care unit. Pediatr Radiol. 2018; 48: 715-721. doi: 10.1007/ s00247-018-4081-4

36. Fernández R, Moreno-Torres M, Contreras AM, Núñez MI, Guirado D, Peñas L. Patient and staff dosimetry during radiographic procedures in an intensive care unit. J Radiol Prot. 2015; 35: 727-732. doi: 10.1088/0952-4746/35/3/727

37. Elshami W, Abuzaid MM, Tekin HO. Effectiveness of breast and eye shielding during cervical spine radiography: An experimental study. Risk Manag Healthc Policy. 2020; 13: 697-704. doi: 10.2147/RMHP.S257185

38. Perdomo A, McMahon S, Wilkie T, Fox N, Rao P. Do carers and comforters require lead aprons during general radiographic examinations? J Med Imaging Radiat Oncol. 2021; In press: 1-9. doi: 10.1111/1754-9485.13304

39. Hayre CM, Bungay H, Jeffery C. How effective are lead-rubber aprons in protecting radiosensitive organs from secondary ionizing radiation? Radiography (Lond). 2020; 26: e264-e269. doi: 10.1016/j. radi.2020.03.013

40. Hung M-C, Chang P. Increased lead concentrations in the hairs of radiographers in general hospitals. Sci Rep. 2021; 11: 236. doi: 10.1038/s41598-020-80721-3

41. Burns KM, Shoag JM, Kahlon SS, et al. Lead aprons are a lead exposure Hazard. J Am Coll Radiol. 2017; 14: 641-647. doi: 10.1016/j.jacr.2016.10.024

42. Shoag JM, Michael Burns K, Kahlon SS, et al. Lead poisoning risk assessment of radiology workers using lead shields. Arch Environ Occup Health. 2020; 75: 60-64. doi: 10.1080/19338244.2018.1553843

43. Abuzaid MM, Elshami W, Shawki M, Salama D. Assessment of compliance to radiation safety and protection at the radiology department. Iranian journal of radiation research (IJRR). 2019; 17: 439446. doi: 10.18869/acadpub.ijrr.17.3.439

44. Mohd Ridzwan SF, Bhoo-Pathy N, Isahak M, Wee LH. Perceptions on radioprotective garment usage and underlying reasons for non-adherence among medical radiation workers from public hospitals in a middle-income Asian setting: A qualitative exploration. Heliyon. 2019; 5: e02478. doi: 10.1016/j.heliyon.2019.e02478

45. Heo Y, Chun H, Kang S, Lee W, Jang T, Park J. Relating factors to wearing personal radiation protectors among healthcare professionals. Ann Occup Environ Med. 2016; 28: 60. doi: 10.1186/s40557016-0144-x

46. Hayre CM, Blackman S, Carlton K, Eyden A. Attitudes and perceptions of radiographers applying lead $(\mathrm{Pb})$ protection in general radiography: An ethnographic study. Radiography (Lond). 2018; 24: e13-e18. doi: 10.1016/j.radi.2017.07.010

47. Chan J, Auffermann W, Jenkins P, Streitmatter S, Duong P-A. Implementing a novel through-glass chest radiography technique for COVID-19 patients: Image quality, radiation dose optimization, and practical considerations. Curr Probl Diagn Radiol. 2022; 51(1): 38-45. doi: 10.1067/j.cpradiol.2020.12.003

48. Rai A, MacGregor K, Hunt B, et al. Proof of concept: Phantom study to ensure quality and safety of portable chest radiography through glass during the COVID-19 pandemic. Invest Radiol. 2020; 56: 135-140. doi: 10.1097/RLI.0000000000000716

49. Brady Z, Scoullar H, Grinsted B, et al. Technique, radiation safety and image quality for chest $\mathrm{X}$-ray imaging through glass and in mobile settings during the COVID-19 pandemic. Phys Eng Sci Med. 2020;43:765-779. doi: 10.1007/s13246-020-00899-8

50. Medial Radiation Practice Board of Australia (MRPBA). Professional capabilities for medical radiation practitioners. Australia. 2020: 1-17.

51. Clement C, Ruehm W, Harrison J, et al. Keeping the IRCP recommendations fit for purpose. $J$ Radiol Prot. 2021; In press: doi: $10.1088 / 1361-6498 /$ ac1611 